\title{
Ultrasound Method of Li-ion Electric Battery Monitoring
}

\author{
Vladimir Gaponov \\ Department of Safety in Technological Processes and \\ Manufactures \\ Don Sate Technical University (DSTU) \\ Rostov-on-Don, Russia \\ e-mail: v.gaponov6591@ yandex.ru \\ Dmitry Kuznetsov \\ Department of Safety in Technological Processes and \\ Manufactures \\ Don Sate Technical University (DSTU) \\ Rostov-on-Don, Russia \\ e-mail: kuznetsovdm@mail.ru
}

\author{
Vitali Dudnik \\ Department of Safety in Technological Processes and \\ Manufactures \\ Don Sate Technical University (DSTU) \\ Rostov-on-Don, Russia \\ e-mail:vvdudnik@mail.ru \\ Nadezda Afanasieva \\ Department of Safety in Technological Processes and \\ Manufactures \\ Don Sate Technical University (DSTU) \\ Rostov-on-Don, Russia \\ e-mail: nadezda.a.afanasyeva@gmail.com
}

\begin{abstract}
During the operation, the efficiency of Li-ion batteries is reduced. Alternative control methods are necessary for a comprehensive assessment. Experiments show ultrasonic signals appearing during the battery charging-discharging. The article is devoted to their registration and analysis.
\end{abstract}

Keywords-component; acoustic emission, Li-ion battery, carbon matrix.

\section{INTRODUCTION}

Electric batteries are widely used in engineering. Much humanity's hope is related to the development of their capabilities, especially in the directions of electric vehicles and the energy accumulation for renewable energy sources. One of the most distributed and effective types of energy storage devices are Li-ion batteries. During the operation process, the efficiency of the batteries is reduced, so users need to check the degradation process. The existing methods of energy storage devices assessment use volt-ampere characteristics. Such method has limited application and accuracy, and thus, cannot be used in some specific engineering problems. Alternative monitoring methods are needed for comprehensive assessment. This article is devoted to the possibilities of Li-ion batteries efficiency control by acoustic emission methods.

\section{THE PROCESSES OCCURRING DURING THE LI-ION BATTERIES CHARGING}

It is known that most $\mathrm{Li}$-ion batteries use carbon materials for the negative electrodes [1-7]. The reason for this is due to the sufficiently large inter-layer distance of graphite lattice. This distance is so large that it is possible to introduce ion reactions in the inter-layer interval. The inclusion reaction (intercalation) of lithium ions into carbon materials is a complex process, the mechanism and kinetics of which are significantly dependent on the structure of the carbon material and the electrolyte. During the discharging of Li-ion battery, there is lithium deintercalation from the carbon material (on the negative electrode) and lithium intercalation into the oxide (on the positive electrode). When the battery is charging, the processes go in the opposite direction. Consequently, there is no metallic (zero-valence) lithium in the whole system, and the processes of discharge and charge lead to the transfer of lithium ions from one electrode to another.

The carbon matrix used as an anode may has an ordered layered structure (natural or synthetic graphite) or a unordered amorphous or partially ordered structure (coke, pyrolysis or mesophasing carbon, carbon black, etc.). Lithium ions during implementation (see Fig.1) expand the layers of the carbon matrix and are located between them, forming intercalates of various structures. A number of authors point out that the specific volume of carbon materials in the processes of intercalation-deintercalation of lithium ions does not change by more than $10 \%$ [1-7]. Such small volume changes allow using the acoustic emission (AE) phenomenon for the study of electrochemical processes.

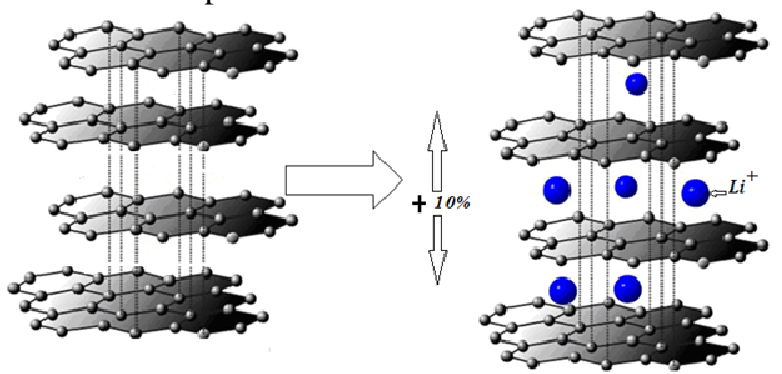

Fig.1. Scheme of lithium ions intercalation into graphite crystal lattice during charging of Li-ion battery. 
As it is known [1-7], during the intercalation of lithium ion in the crystal lattice of graphite as a result of electrochemical processes, the layers of graphite are sliding. Thus, stresses in the lattice structure arise. The deformation appeared in the dislocation shifts of the crystal lattice is accompanied by the generation of high-frequency stress waves (much higher than $1 \mathrm{MHz}$ ). The multiplicity of this process leads to the appearance of the "beating" phenomenon and the generation of low-frequency waves (up to $1 \mathrm{MHz}$ ). The phenomenon of "beating" appears when harmonic oscillations with nearest frequencies are combined. It causes acoustic waves. The amplitude of these waves can exceed the threshold range of $\mathrm{AE}$ devices.

Dynamic volume changes, recorded by piezosensor in the ultrasonic frequency range, are a classical $\mathrm{AE}$ phenomenon widely used in flaw detection [8-10]. Experiments described below were carried out to assess the possibilities of monitoring the charging process in Li-ion batteries.

\section{EXPERIMENTAL EQUIPMENT}

An AE device, which allows checking the signals of the Li-ion battery, is shown in Fig. 2. The Li-ion battery is rigidly connected to the piezosensor, which produces an electrical signal. This signal passed through the amplifier and entered the complex A-line 32D, where it was recorded. At the same time, the volt-ampere characteristics of the electrical network were monitored on the battery connection line. The battery could be connected to both the electric load and the charging device.

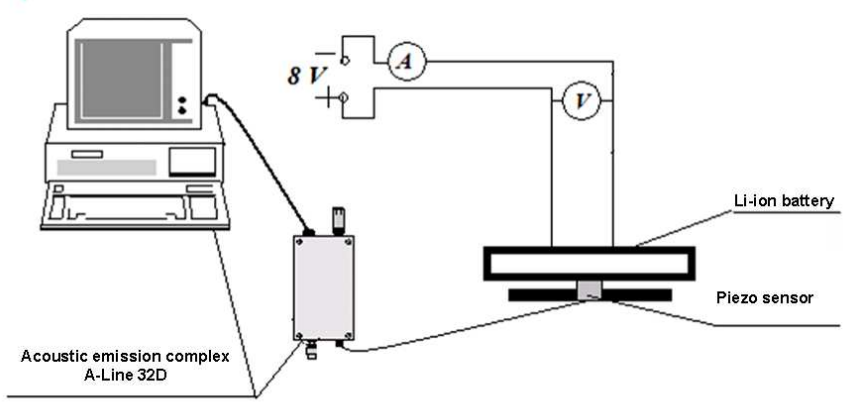

Fig. 2. The schematics of the experimental complex for measuring the electrical parameters and $\mathrm{AE}$ impulses during the charging of a lithium-ion battery.

The electric current and voltage on the battery were recorded during the experiments. Simultaneously with the registration of electrical parameters of the electrolysis process, the characteristics of the induced ultrasonic acoustic signals were recorded. There were the following parameters (Fig. 3):

- emission amplitude - the maximum value of the emission signal for a time period;

duration of the electrical signal (sec) - the time of the envelope of the electric impulses from $\mathrm{AE}$ above the threshold of the restriction.
- $\quad$ rise time (sec) - the time when the envelope curve of the AE impulse rise over the threshold of the restriction,

- total counts (signals) - the number AE impulses over the threshold of the restriction.

ejections (signals) - the number of oscillations exceeding the established level of discrimination during the envelope period of the electric impulses from the $\mathrm{AE}$ over the threshold of the restriction.

AE count.

activity (signal $/ \mathrm{sec}$ ) - the time derivative of the total

- $\quad$ energy of the electrical signal $(\mathrm{J})$ - the area under the envelope of the electrical signal from AE.

First of all, the amplitude, activity and duration of the signal were subjected to analysis.

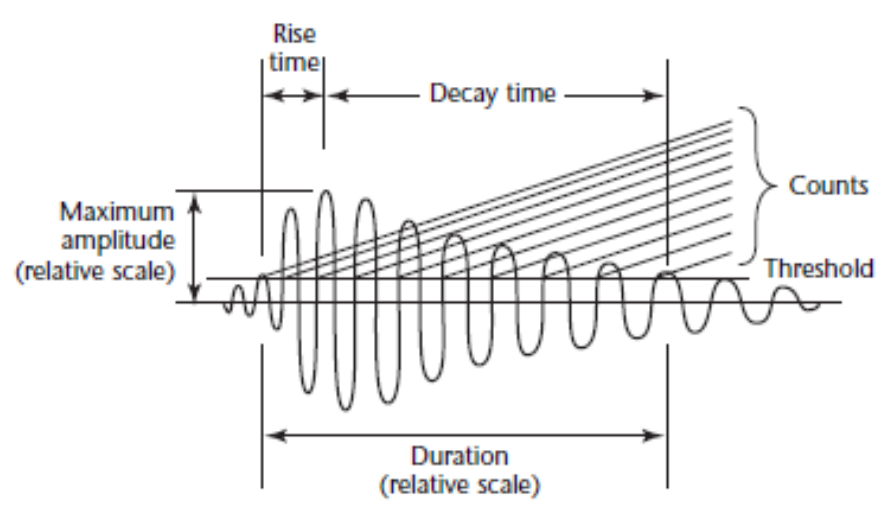

Fig.3. Recorded data of the ultrasonic acoustic signal.

\section{EXPERIMENTAL RESULTS}

The first part of the experiments was devoted to the recording of the processes that occur during the batteries charging. In the process of charging, voltage is increased asymptotically approaches to the maximum value (Fig. 4). The AE signals activity also changes during charging of Li-ion batteries as can be seen from the obtained data. This change corresponds to the dynamics of the voltage change.

In the first 200-300 seconds of the charging, the voltage is growing fast from 7.551 to $7,758 \mathrm{~V}$ (change is $2.7 \%$ ). The $\mathrm{AE}$ activity in the same period is dropping from the values of 130160 signals/sec to the $60-90$ signals/sec (change is about $50 \%$ of the measured value). Consequently, the initial stage of the charging process is sufficiently reliable fixed by $\mathrm{AE}$ parameters. 


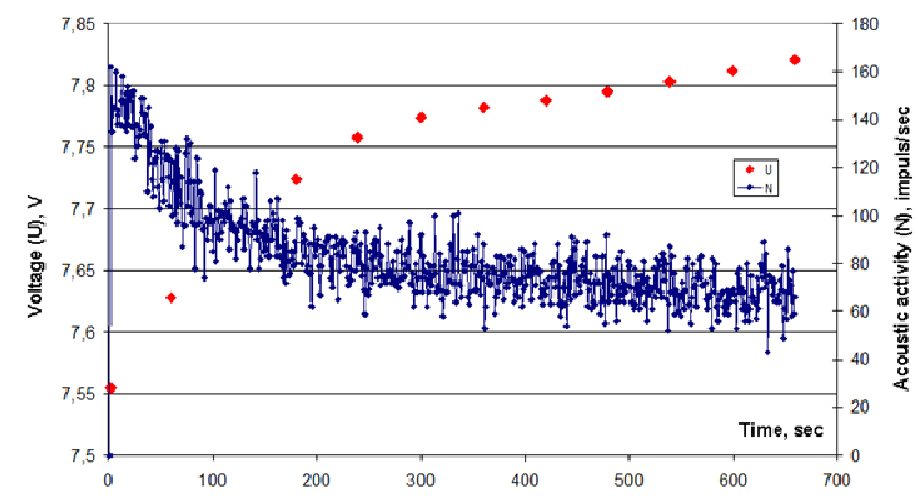

Fig.4. Changes in voltage (V) and AE activity (signals/sec) during the charging of a lithium-ion battery.

The interpretation of the obtained data may be as follows. In the process of charging a fully discharged Li-ion battery, the intercalation of lithium ions causes sharp increasing in the negative electrode volume. Graphite is a material with a low modulus of elasticity. It can be suggested that, although the interlayer distance in the graphite grid increases all the time as Li-ion batteries are charged, the rate of this increasing reduces.

The plasticity of graphite is weak at the macro-crystalline level; however, at the level of the crystal lattice, the plasticity phenomenon can be realized in reducing the linear changes rate in the interlayer distances of the graphite lattice. The reason for it is the low value of the elastic modulus of graphite. The initial stage of charging should be characterized by the highest rate of linear changes of interlayer distances of the graphite lattice. Such changes were registered by means of AE parameters.

Interestingly, when the charged battery is turned on to the charging mode, the sharp surge of acoustic signals is not observed.

Recording of parameters during battery discharge showed that when the battery is switched on to the discharge mode, there is always a surge of acoustic emission signals at the first moments of time. Then it is reduced. This surge is highly dependent on the battery temperature. Fig. 5 shows the record of $\mathrm{AE}$ activity during the repetition of the charge/discharge battery cycles. The duration of ultrasonic signals changes together with AE activity. Despite the fact that the dependence of the signal duration curve does not repeat the activity dependence, it shows also a surge of signals when the discharge mode is switched on. Thus, at this moment of time not only the number of impulses increases, but also their duration time does. It means that the energy of the signals grows many times as much. The charged battery during the experiment was switched from charge to discharge. You can see that the charge of the battery does not increase the activity of emission in this case.

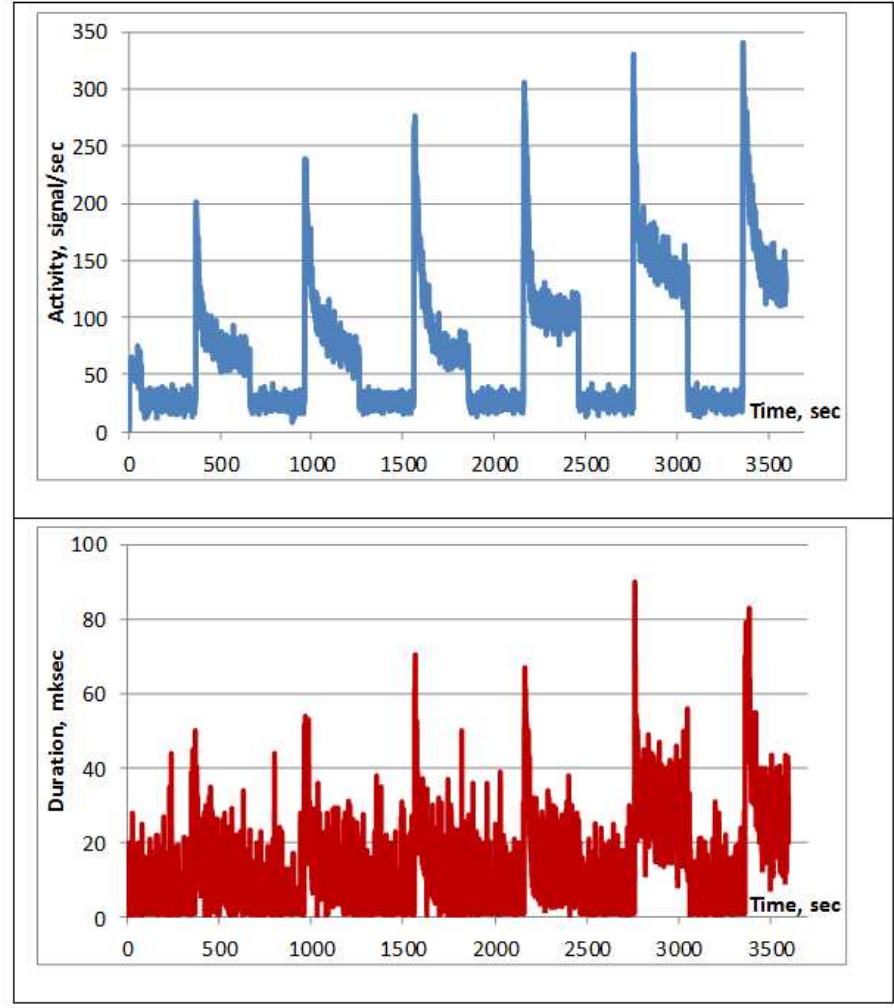

Fig.5. AE activity (top) and duration (bottom) during the cyclic changing of charge/discharge modes.

When the electric load is connected to battery, the discharge begins and a sharp surge of signals is observed always. Then it gradually decreases. Detailed recording of AE in the discharge mode during the first and sixth discharge interval with a difference of 50 minutes is shown in Fig. 6. The experiment showed that the heating occurring during the battery operation increases the activity of acoustic emission signals in the moment of switching on. Thus, in the process of cyclic switching, the value of maximum activity increased from 201 signals/sec to 340 signals/sec (TABLE 1). For each discharge cycle observed during the experiment, an approximation function, also presented in Table 1, was determined. It allowed determining the dynamics of $\mathrm{AE}$ activity in all discharge cycles and describing it by the following general equation:

$$
N=A \operatorname{Ln}(t)+C
$$

where $N$ - AE activity (signal/sec),

$t$ - discharge time (sec),

$A$ and $C$ - empirical coefficients. 


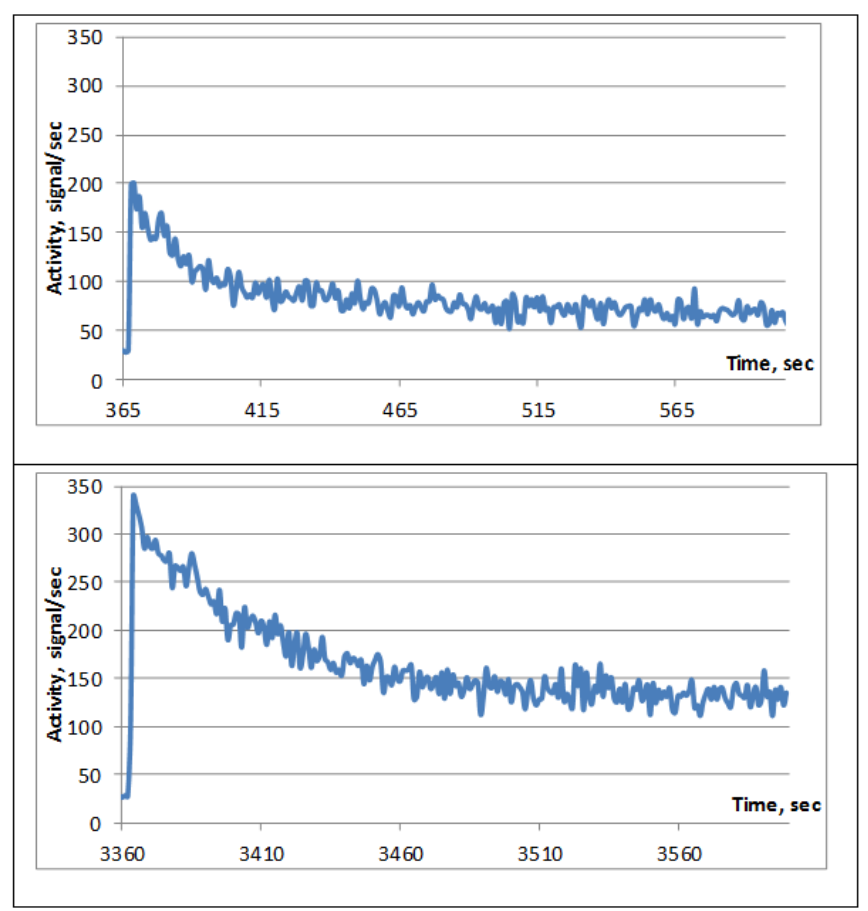

Fig.6. AE activity during the battery discharge mode at the beginning (top) and at the end (bottom) of the experiment.

TABLE I. CHANGING OF AE ACTIVITY DURING THE DISCHARGING OF LI-ION BATTERIES.

\begin{tabular}{|l|l|l|}
\hline $\begin{array}{c}\text { № } \\
\text { cycle }\end{array}$ & $\begin{array}{c}\text { Equation of AE activity } \\
\text { change depending on time }\end{array}$ & $\begin{array}{c}\text { Maximum value } \\
\text { of AE activity, N, } \\
\text { signals/sec }\end{array}$ \\
\hline 1 & $\mathrm{~N}=-23.51 \mathrm{Ln}(\mathrm{t})+191.08$ & 201 \\
\hline 2 & $\mathrm{~N}=-36.618 \mathrm{Ln}(\mathrm{t})+265.9$ & 239 \\
\hline 3 & $\mathrm{~N}=-44.836 \mathrm{Ln}(\mathrm{t})+307.15$ & 276 \\
\hline 4 & $\mathrm{~N}=-38.299 \mathrm{Ln}(\mathrm{t})+299.05$ & 306 \\
\hline 5 & $\mathrm{~N}=-30.068 \mathrm{Ln}(\mathrm{t})+297.8$ & 330 \\
\hline 6 & $\mathrm{~N}=-44.66 \mathrm{Ln}(\mathrm{t})+366.68$ & 340 \\
\hline
\end{tabular}

More detailed analysis of changes in the dynamics of $\mathrm{AE}$ impulses, observed in each charge-discharge cycle, showed that not only the maximum value of AE activity increases. The sum of $\mathrm{AE}$ impulses registered for each cycle increases as well. For clarity, Fig. 7 shows the growth curve of the AE impulses sum recorded during the entire experiment. The curve clearly shows not only individual waves of the sum of $\mathrm{AE}$ impulses, characteristic for each charge-discharge cycle, but also the general trend of change. The time dependence of the sum of the impulses describes a non-linear law, which should be expected in the case of the system returning to the initial state during the charge-discharge cycle. But the system has a gradual deviation of the state from the equilibrium situation.

The growth curve of the number of signals with time is described by a polynomial curve with a confidence value of 0.9964. It is indicative not of stochastic nature of the changing, but of pronounced functional dependence. Interpretation of $\mathrm{AE}$ data in this case may be as follows: the recorded ultrasonic signals indicate the gradual accumulation of damages in the carbon matrix signals as the chargedischarge cycles are carried out.

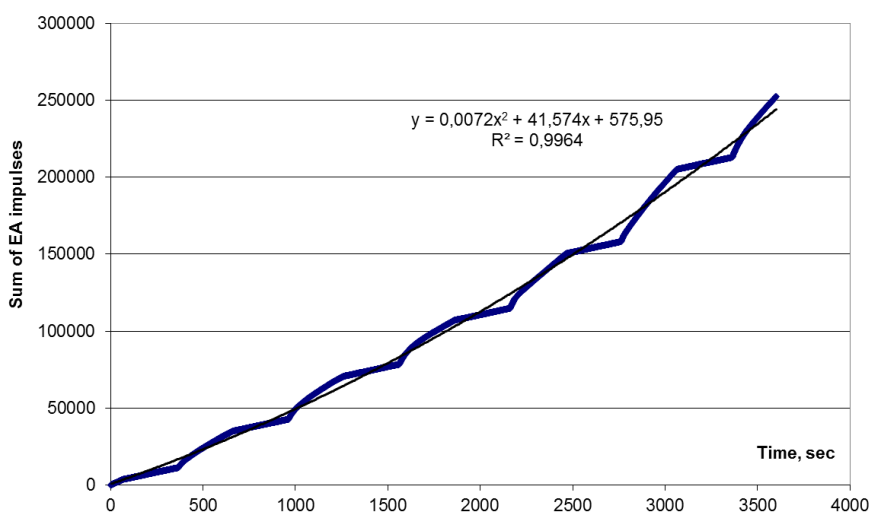

Fig. 7. The sum of the AE impulses recorded during the entire experiment.

The dynamics of the AE signals changing (the sum of signals) in time allows one to offer general provisions based on the assumption that the activity of $A E(N)$ signals, induced during the charging, is proportional to the concentration difference $(\Delta C)$ of intercalated lithium ions $\mathrm{C} 1$ in the carbon structure of the matrix and free lithium ions $\mathrm{C} 3$ :

$$
N \approx f(\Delta C)
$$

Accordingly, the activity of $\mathrm{AE}$ signals is proportional to the rate of the concentration difference $\Delta \mathrm{C}$ :

$$
\frac{d N}{d t}=K \frac{d \Delta C}{d t}
$$

where $\mathrm{K}$ - proportionality factor.

In the initial moment, the concentration difference is equal to $\Delta C_{0}$. At time $(t)$, the number of intercalated lithium ions $C 1$ is equal to difference $\left(\Delta C_{0}-\Delta C\right)$. The rate of changing of the concentration difference is equal to $\frac{d \Delta C}{d t}$.

Hence, we obtain the following differential equation of the process:

$$
\frac{d \Delta C}{d t}=k\left(\Delta C_{0}-\Delta C\right)
$$

Integrating this equation, we obtain a solution relatively the concentration difference $(\Delta C)$ of intercalated and not yet intercalated $\mathrm{Li}$-ions $\mathrm{C} 1$ under the initial condition of $\mathrm{t}=0$ :

$$
k \Delta C_{0}-\Delta C=k \Delta C_{0} e^{-t / \tau}
$$




$$
\Delta C=\Delta C_{0}\left(1-e^{-t / \tau}\right)
$$

where $\tau$ is the relaxation time constant, which depends on the intercalation rate of lithium ions and depends on the type of battery, the type of carbon matrix (soot, graphite, disordered carbon), and the geometric parameters of the cathode and anode.

The general equation of the total number of AE signals changing during the charging can be obtained using equations (2) and (6):

$$
N=K_{1} \Delta C_{0}\left(1-e^{-t / \tau}\right)
$$

where $K_{1}$ is a coefficient that depends on the method of acoustic signals recording and the sensitivity of the acoustic emission equipment.

Analyzing the dynamics of the total AE count during the charging a fully discharged Li-ion battery, the expected curve was obtained (Fig. 8):

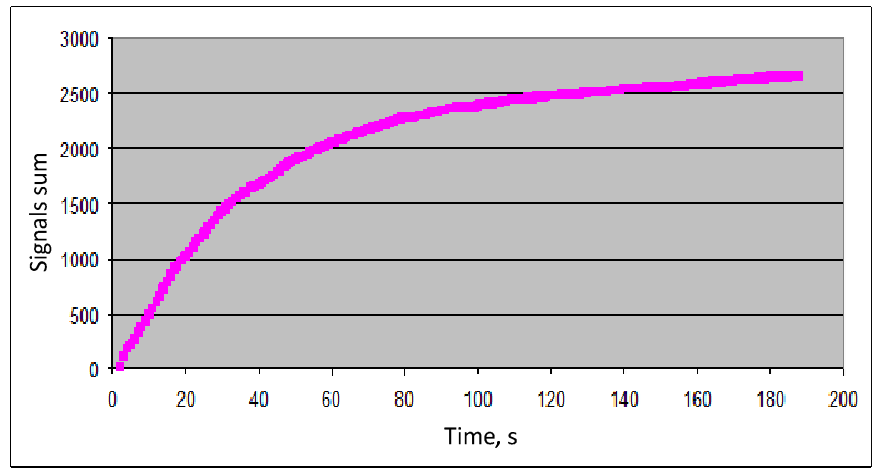

Fig.8. Changing of the signals sum during the charging a fully discharged Liion battery.

At the same time, the informativeness of the proposed AE method is such that it is possible to evaluate the magnitude of the accumulated damage. The AE method uses the value of the AE signal amplitude, as one of the main recorded parameters. It acts often as the main parameter of the defect magnitude. During the experiments, it was found that, despite the increase in the maximum activity of the signals and the sum of the $\mathrm{AE}$ signals for each cycle, an increase in the AE signals amplitude was no recorded. It means that the processes of intercalationdeintercalation of lithium ions in the carbon matrix at this stage have not led to a disruption of the continuity of the material and to the appearance of additional dislocations or defects. Any violation of the continuity of the carbon material during expansion (intercalation of lithium ions) or shrinkage (deintercalation of lithium ions) should lead to friction of microcrack banks or their development. These processes are always accompanied by an increase of the AE signals amplitude and are often identified precisely by the phenomenon of $\mathrm{AE}$ as the most sensitive method of flaw detection.

\section{CONCLUSION}

In conclusion, the following important results should be noted from the experimental study:

- ultrasonic vibrations are generated at any battery charge/discharge cycle. It can be detected by AE equipment;

- when a weakly charged battery switched to the charge mode a sharp rise of $\mathrm{AE}$ activity is observed during the starting stage;

- when a battery is charged enough, the sharp rise of $\mathrm{AE}$ activity is not observed at the starting stage of the charge process;

- when a battery is switched to the discharge mode, the sharp rise of AE activity also appears during the starting stage. At further stages, AE activity decreases logarithmically.

The further investigations will allow developing a methodology of batteries analysis and assessing, based on the phenomenon of acoustic waves generation during the electrochemical reactions in lithium ion batteries. Experiments also allow admitting that other battery types can also be controlled by the AE method.

\section{REFERENCES}

[1] L. Giordano, P. Karayaylali, Y. Shao-Horn, Y. Yu, Y. Katayama, F. Maglia, "Chemical Reactivity Descriptor for the Oxide-electrolyte Interface in Li-ion Batteries", Journal of Physical Chemistry Letters, vol. 8, 2017, pp. 3881-3887.

[2] A. Ott, P. Endres, V. Klein, B. Fuchs, A. Jager, H. Mayer, "Electrochemical performance and chemical properties of oxidic cathode materials for $4 \mathrm{v}$ rechargeable li-ion batteries", Journal of Power Sources, vol. 1, 1998, pp. 1-8.

[3] O. Bergstrom, H. Bjork, T. Gustafsson, J. Thomas, "Direct xrd observation of oxidation-state changes on li-ion insertion into transitionmetal oxide hosts", Journal of Power Sources, vol. 81, 1999, pp. 685689.

[4] F. Ning, S. Li, B. Xu, C. Ouyang, "Strain tuned li diffusion in licoo2 material for li ion batteries: a first principles study", Solid State Ionics, vol. 263. 2014, pp. 46-48.

[5] M. Endo, C. Kim, K. Nishimura, T. Fujino, K. Miyashita, "Recent development of carbon materials for li ion batteries", Carbon, vol. 2, 2000, pp. 183-197.

[6] C. Gong, M. Acik, Y. Chabal, K. Cho, R. Abolfath, "Graphitization of graphene oxide with ethanol during thermal reduction", Journal of Physical Chemistry C, vol. 18, book. 116, 2012, pp. 9969-9979.

[7] C. Lu, L. Zhang, J. Ma, Z. Chen, L. Tao, Y. Su, "Li-ion battery capacity cycling fading dynamics cognition: a stochastic approach", Energy, vol. 137, 2017, pp. 251-259.

[8] S.I. Builo, D.M. Kuznetsov, "Acoustic-emission testing and diagnostics of the kinetics of physicochemical processes in liquid media", Russian Journal of Nondestructive Testing, vol. 9, 2010, pp. 684-689.

[9] D.M. Kuznetsov, "Shrinkage phenomena in graphitization of preforms in castner furnaces", Refractories and Industrial Ceramics, vol. 7-8, 2000, pp. 279-282.

[10] V.A. Barat, D.V. Chernov, S.V. Elizarov, "Discovering data flow discords for enhancing noise immunity of acoustic-emission testing", Russian Journal of Nondestructive Testing, vol. 6. 2016, p. 347-356. 